\title{
The Mechanism of Thermomechanical Training of a Newly Developed Fe-Mn-Si-Cr-Cu Shape Memory Alloy
}

\author{
D.P. Dunne and H. Li \\ Department of Materials Engineering, University of Wollongong, Wollongong, NSW 2522, Australia
}

\begin{abstract}
Thermomechanical training was carried out on a newly developed $\mathrm{Fe}-20 \mathrm{Mn}-6 \mathrm{Si}-7 \mathrm{Cr}-1 \mathrm{Cu}$ shape memory alloy. It was observed that the effectiveness of thermomechanical training largely depends on the recovery annealing temperature and the pre-strain during training. Based on the analysis of the changes in critical stress for martensite formation, the critical stress for slip deformation, the phase transformation temperatures and microstructural development during training, it was concluded that the mechanism of thermomechanical training is based on the following two factors: (1) lowering of the critical stress for martensite formation, making stress induced $\gamma \rightarrow \varepsilon$ transformation easier; and (2) decreasing the Af temperature and refining the $\varepsilon$ martensite structure, thus reducing the energy barrier for the $\varepsilon \rightarrow \gamma$ reverse transformation. The optimum thermomechanical training conditions have been determined for the alloy studied.
\end{abstract}

\section{INTRODUCTION}

It has been reported[1-5] that thermomechanical training can significantly improve the shape memory effect of Fe-Mn-Si based alloys. The proper training process can suppress slip deformation by strengthening of the austenitic matrix, and can lower the stress for inducing $\varepsilon$ martensite by creating dislocation structures which are favourable to martensitic transformation. However, the mechanism of thermomechanical training remains unclear. This paper reports experimental results, presents discussion on the factors influencing thermomechanical training and explains the mechanism of training through the analysis of mechanical characteristics and microstructure.

\section{EXPERIMENTAL}

The selected alloy $\mathrm{Fe}-20 \mathrm{Mn}-6 \mathrm{Si}-7 \mathrm{Cr}-1 \mathrm{Cu}$ (wt\%) was produced by vacuum melting. The $6 \mathrm{~kg}$ ingot was annealed at $1473 \mathrm{~K}$ for 1 hour, and then hot rolled to plate $7 \mathrm{~mm}$ in thickness. Tensile test samples ( $3 \mathrm{~mm}$ dia X 40mm) were machined from the plate, and austenitized at $873 \mathrm{~K}$ for $30 \mathrm{~min}$. The martensite and austenite transformation temperatures (Ms, As and Af) were determined by DSC and TMA. In the current study, thermomechanical training was carried out using tensile straining. The thermomechanical training schedule consisted of tensile straining within the range from $2 \%$ to $6.5 \%$, followed by heating to a selected temperature in the range from $673 \mathrm{~K}$ to $1073 \mathrm{~K}$ for 10 minutes, then quenching in hot water. Optical and transmission electron microscopy were used to study the microstructures of the alloy after selected treatments.

\section{RESULTS AND DISCUSSION}

\subsection{The effect of training on shape memory effect}

Figure 1 shows the changes of recovery ratio of the alloy with increasing thermomechanical training cycles for training strains of $2 \%$ and $4 \%$ and thermal treatment temperatures in the range $673-973 \mathrm{~K}$. These data show that the investigated alloy has a good shape memory in the untrained state ( 90\%) and that $100 \%$ 
shape recovery can be easily obtained for a $2 \%$ training strain. In the case of $4 \%$ training strain, recovery annealing at medium temperatures (from $773 \mathrm{~K}$ to $873 \mathrm{~K}$ ) was most effective for the improvement of shape memory effect. Nearly complete shape recovery was obtained after 4 training cycles, and then the recovery ratio remained constant with increasing training cycles. When the recovery annealing temperature was $673 \mathrm{~K}$, the shape recovery ratio decreased dramatically with increasing training cycles for $4 \%$ training strain. This phenomenon suggests that unrecovered structure induced by training accumulates with increasing number of training cycles, and adversely affects the $\gamma \leftrightarrow \varepsilon$ transformation.
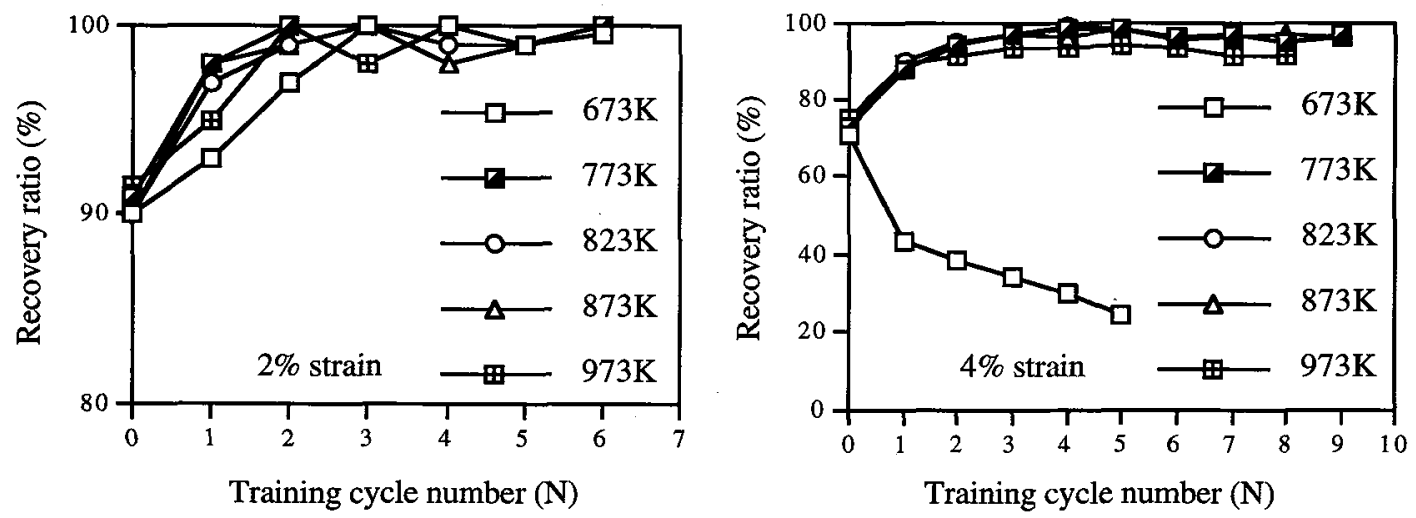

Figure 1: The effect of thermomechanical training on the shape memory effect (2\% and $4 \%$ training strain)

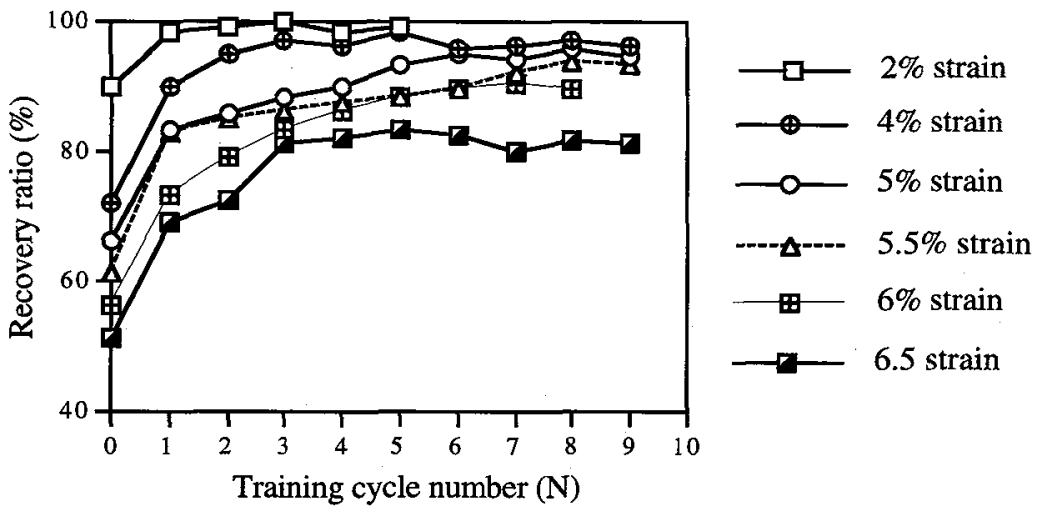

Figure 2: The effect of training strain on the shape recovery ratio for recovery annealing at $873 \mathrm{~K}$ for $10 \mathrm{mins}$.

The effectiveness of training is sensitive to the recovery annealing temperature. Based on the results, annealing at $873 \mathrm{~K}$ was selected for further study. The effect of training strain on thermomechanical training is shown in Figure 2 for a recovery annealing temperature of $873 \mathrm{~K}$; the recovery ratio gradually decreased with increasing training strain. Since the recovery strain is the most important factor in commercial applications, Figure 3 is a plot of the changes in recovery strain with increasing training cycles for different training strains. It is clear that recovery strain increases with increasing training strain, and 
saturates at about $6 \%$ training strain, with the maximum recovery strain being about $5.4 \%$. However, for untrained samples (initial state), the recovery strain appears to reach a saturation value at about $3.3 \%$. This suggests that the most effective thermomechanical training strain is about $6 \%$.

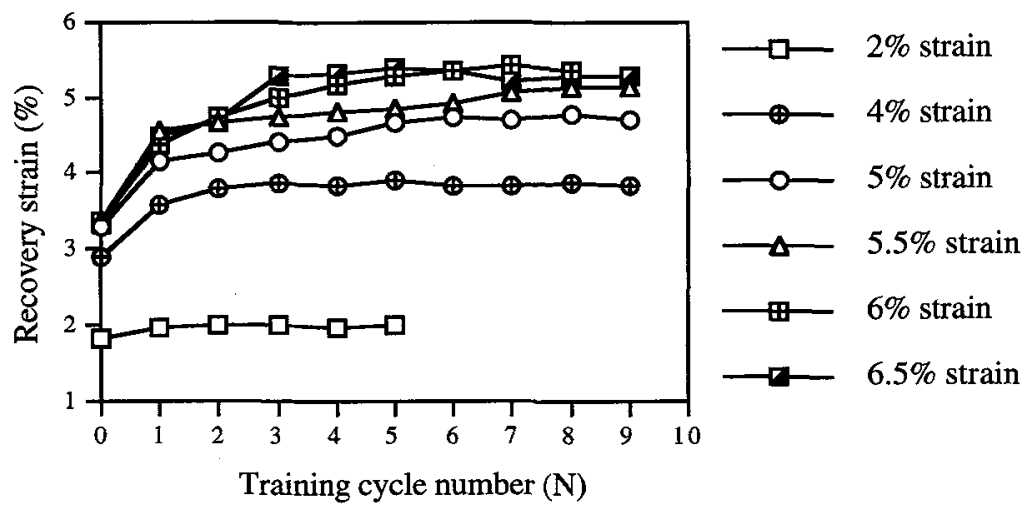

Figure 3: The effect of training strain on recovery strain after thermomechanical training with training strain in the range 2$6.5 \%$ and a recovery annealing temperature of $873 \mathrm{~K}$

\subsection{The effect of training on phase transformation temperatures}

The present data indicate that an appropriate training process can significantly enhance the shape memory effect in Fe-Mn-Si based alloys studied. Since the shape memory effect is governed by $\gamma \leftrightarrow \varepsilon$ transformation, the effect of thermomechanical training on transformation is critical.

The transformation temperatures (Ms, As and Af) directly reflect the characteristics of $\gamma \leftrightarrow \varepsilon$ transformation. Figure 4 illustrates the changes of $\mathrm{Ms}$, As and Af with increasing training cycles for a training strain of $4 \%$. It is clear that while the Ms and As temperatures remain constant with increasing number of training cycles, the Af temperature is influenced by training. For a recovery annealing temperature of $673 \mathrm{~K}$, Af temperature increases with increasing number of training cycles; for example, the increase was nearly $70 \mathrm{~K}$ after five cycles ( from $647 \mathrm{~K}$ to $716 \mathrm{~K}$ ). The Af temperature became higher than the recovery annealing temperature after 3 training cycles, and it is inferred that the accumulation of unrecovered structure causes obstacles to the reverse motion of Shockley partial dislocations, making $\varepsilon \rightarrow \gamma$ transformation more difficult and leading to degradation of the shape memory effect. In the case of the recovery annealing temperature being higher than $773 \mathrm{~K}$, Af temperature decreased with increasing training cycle number and appeared to reach saturation after 5 to 6 training cycles. Since Af decreased by nearly $20 \mathrm{~K}$, it can be concluded that the dislocation structure created by training is favourable to reverse transformation, leading to an improvement in shape memory effect.

\subsection{The effect of training on mechanical behaviour}

Because stress induced $\gamma \rightarrow \varepsilon$ transformation is critical for shape memory, the mechanical behaviour is related to the transformation behaviour. In the current study, the mechanical behaviour of the alloy was studied by tensile testing.

Stress induced martensitic transformation is largely dependent on the deformation temperature. If the deformation temperature is higher than the Md temperature, $\gamma \rightarrow \varepsilon$ transformation will be totally replaced by slip deformation. To obtain a good shape memory, it is important to suppress slip deformation. The critical stress for martensite formation and that for slip deformation are functions of temperature, with the former showing positive temperature dependence and the latter a negative dependence. The magnitudes of these two stresses become equal at a certain temperature ( $\left.\mathrm{T}^{\prime}\right)$. These stresses can be approximated by the measured yield stress in a tensile test and the temperature dependence of yield stress is shown in Figure 5. 
The critical stress for martensite formation and that for slip deformation correspond to the yield stress for $\mathrm{T}<\mathrm{T}^{\prime}$ and $\mathrm{T}>\mathrm{T}^{\prime}$, respectively. It is evident that in order to prevent slip deformation, the deformation temperature must be lower than T'.
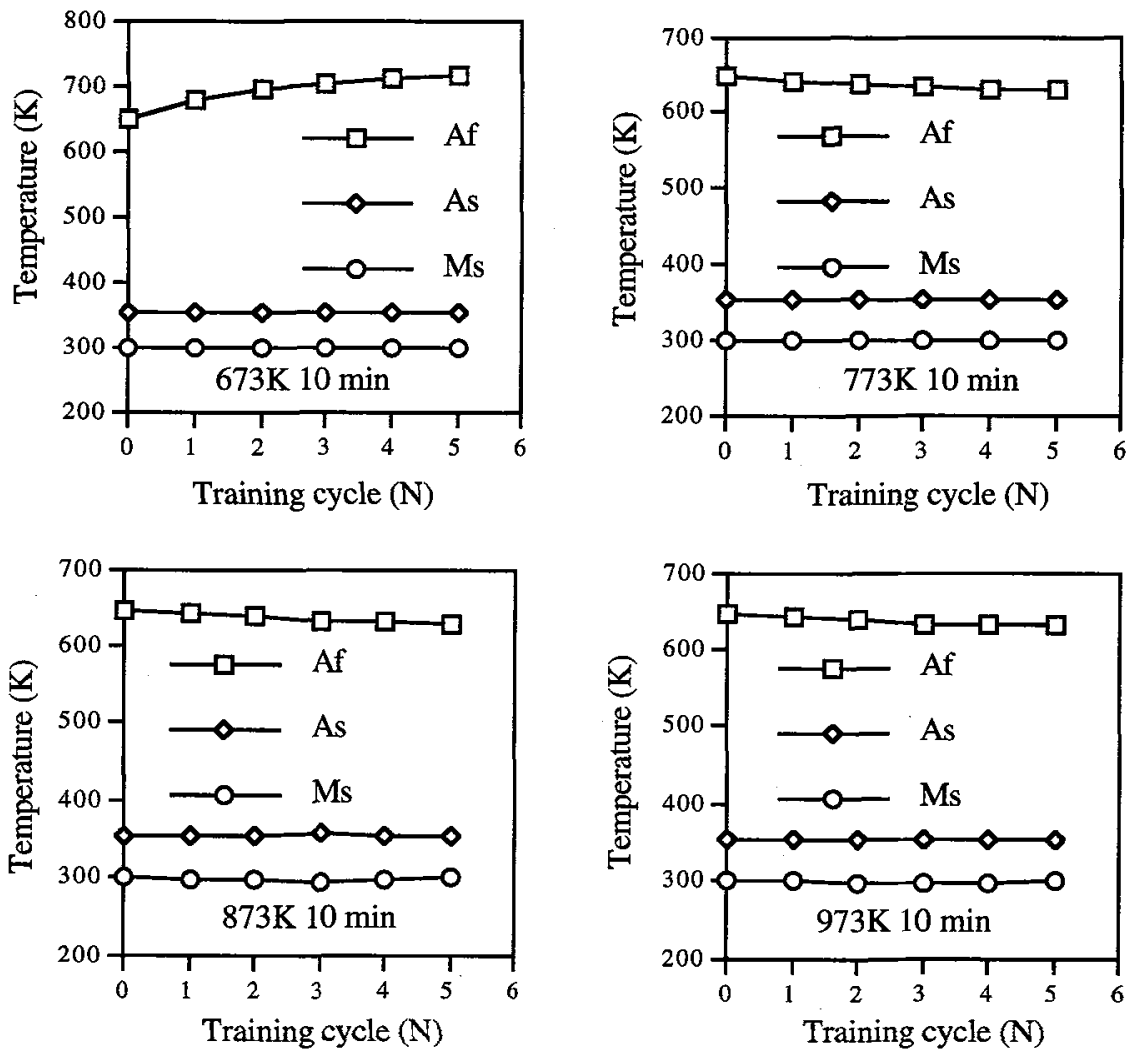

Figure 4: The effect of thermomechanical training (4\% training strain) on phase transformation temperatures as a function of training cycle number

The influence of thermomechanical training on the yield stress has been investigated in the current study. Figure 6 gives the critical stresses for martensite formation and for slip deformation at room temperature and 473K. Figure 6 (a) shows the change in yield stress with increasing training cycle number at room temperature for a training strain of $4 \%$. It is evident that the change in yield stress largely depends on the recovery annealing temperature. When the recovery annealing temperature is $673 \mathrm{~K}$, the yield stress increases with increasing cycle number, which means that the critical stress for martensite formation increases, leading to the degradation of shape memory effect. When the recovery annealing temperature is in the range of $773 \mathrm{~K}$ to $973 \mathrm{~K}$, the critical stress for martensite formation decreases with increasing cycle number, and approaches a saturation value. The decrease in critical stress for martensite formation is considered beneficial to stress induced $\gamma \rightarrow \varepsilon$ transformation, leading to an improvement in the shape memory effect. On the basis of these results, annealing at $873 \mathrm{~K}$ appears to be the most efficient recovery temperature. The beneficial effect of training decreases when the recovery annealing temperature is increased to $973 \mathrm{~K}$, suggesting that the dislocation structure created by training, which is favourable to the formation of stress induced martensite, is eliminated or strongly modified by restoration during annealing.

Figure 6 (b) illustrates the changes of yield stresses at $473 \mathrm{~K}$ with increasing number of training cycles for a training strain of $4 \%$. The yield stress at $473 \mathrm{~K}$ (which is higher than the Md temperature) reflects the critical stress for slip deformation (strength of the matrix). The yield stress remains almost constant with increasing training cycles when the recovery annealing temperature is $823 \mathrm{~K}$, but the austenitic matrix is 
strengthened when the recovery annealing temperature is lower than $773 \mathrm{~K}$, and slightly softened when the recovery annealing temperature is higher than $873 \mathrm{~K}$. Although the effect of thermomechanical training on the parent phase strength depends on the recovery annealing temperature, the effect is not as marked as for the stress to induce martensitic transformation (Figure 6(a)).

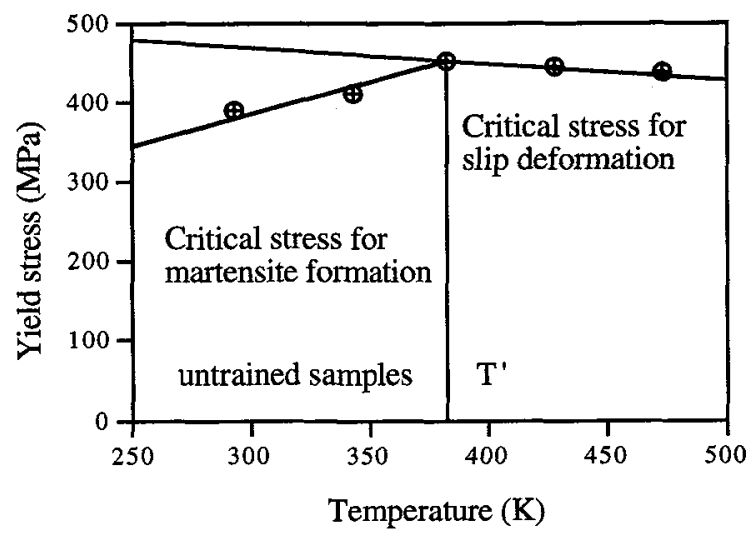

Figure 5: Temperature dependence of yield stress (for untrained samples)
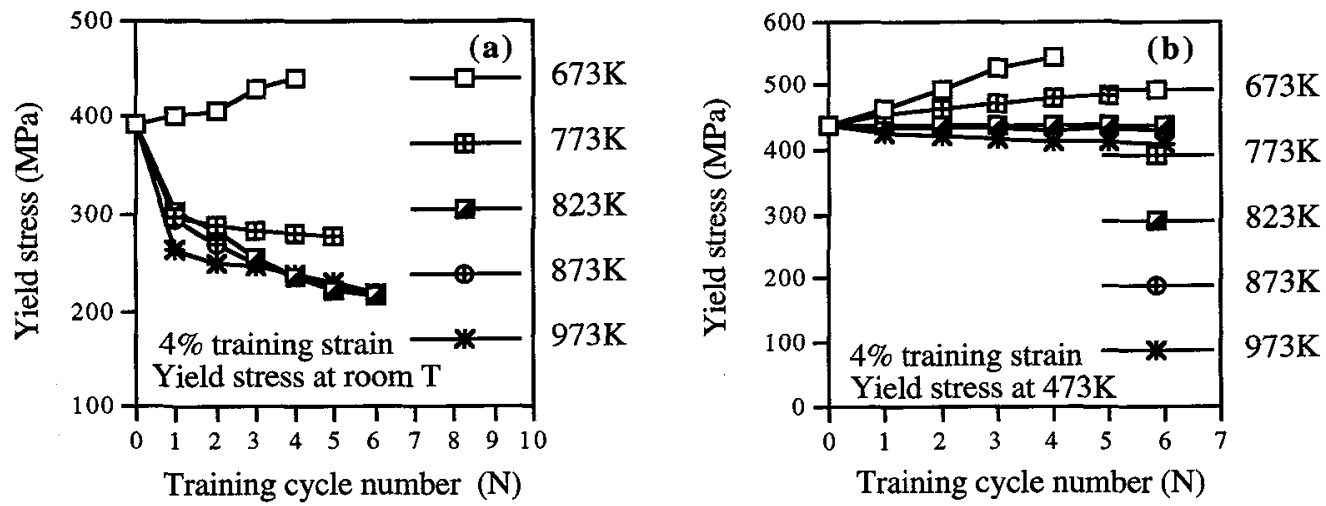

Figure 6: The effect of training on yield stress of the alloy (a) at room temperature ( $\left.<\mathrm{T}^{\prime}\right)$ and (b) at $473 \mathrm{~K}\left(>\mathrm{T}^{\prime}\right)$

Figure 7 shows the effect of training on the temperature dependence of yield stress (4\% training strain and 4 cycles). It is clear that the parent phase strength is not markedly temperature dependent when the recovery annealing temperature is between $773-973 \mathrm{~K}$, but stress to induce martensite is highly sensitive to test temperature. These results confirm that the improved shape memory associated with training flows from the reduction in the critical stress for $\varepsilon$ martensite formation rather than strengthening of the austenitic matrix.

\subsection{Metallographic features}

The shape memory effect in Fe-Mn-Si based shape memory alloys can be improved by thermomechanical training, suggesting that the training process influences stress induced $\gamma \rightarrow \varepsilon$ transformation and its reverse transformation. The effect of thermomechanical training on transformation was investigated by observing the microstructures of selected samples. Figure 8 shows the microstructures of samples before training and after training ( 4 training cycles, $4 \%$ training strain, and $873 \mathrm{~K}$ recovery temperature). It was found that 
the thickness of the $\varepsilon$ martensite plates decreased after thermomechanical training, whereas the amount measured optically and by $\mathrm{X}$-ray diffraction, did not show any significant change.

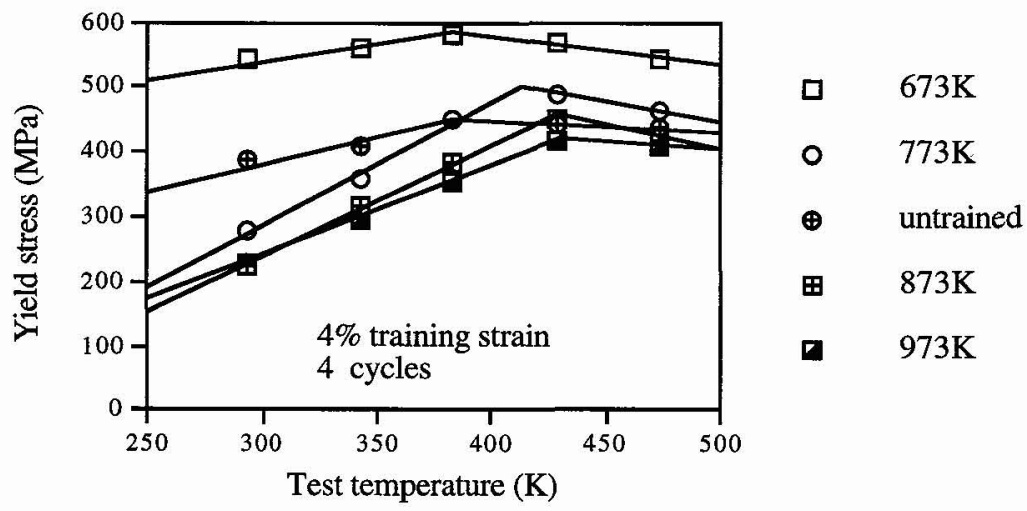

Figure 7: The effect of thermomechanical training on the temperature dependence of yield stress
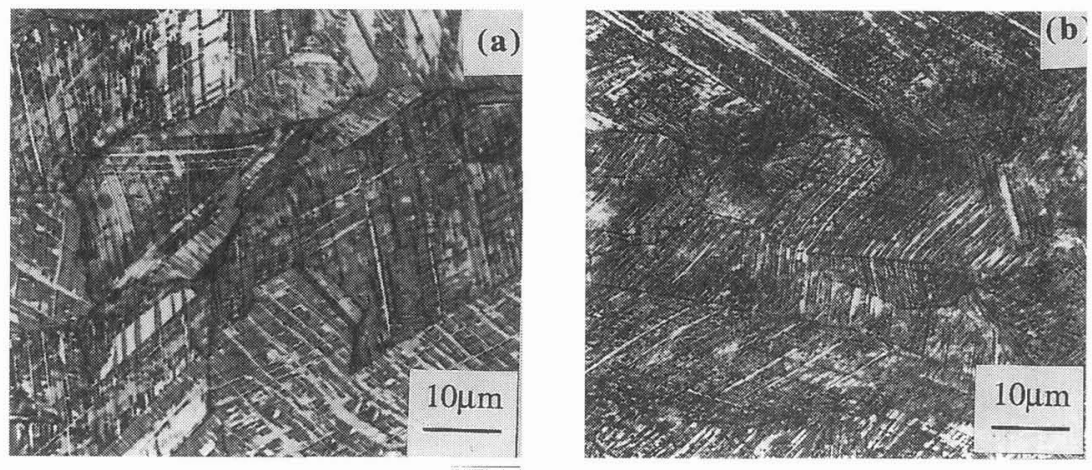

Figure 8: The microstructures of an untrained sample (left) and a trained sample (4\% pre-strain)(right)

\section{CONCLUSIONS}

Based on these results, two main conclusions can be drawn. Firstly, the kinetics of $\gamma \rightarrow \varepsilon$ transformation are influenced by thermomechanical training, with the density of nucleation sites for $\varepsilon$ martensite in trained samples being higher than for untrained samples, ie., the nucleation rate of stress induced martensite in trained samples is higher, with the energy barrier to nucleation being lower. Secondly, $\varepsilon \rightarrow \gamma$ reverse transformation becomes easier after training because thinner martensite plates cause smaller local shape strains. These two effects are considered to result in the observed improvement in shape memory capacity.

\section{References}

[1] H. Otsuka, M. Murakami and S. Matsuda, "Improvement of the Shape Memory Effect of Fe-Mn-Si Alloys by Thermomechanical Treatment ", Proc. of the MRS International Conference on Advanced Materials, Tokyo, Japan, May 31-June 3, 1988. M. Doyama, S. Somiya and R. P. H. Chang, eds. (Materials Research Society, Pittsburgh, Pennsylvania, 1989), Volume 9, pp. 451-456.

[2] J. S. Robinson and P. G. McCormick, Scripta Metall., Volume 23 (1989), 1975-1978.

[3] X. X. Wang and L. C. Zhao, Scripta Metall., Volume 26 (1992), 1451-1456.

[4] H. Inagaki, Z. Metallkd, Volume 83 (1992) 90-104.

[5] Y. Watanabe, Y. Mori and A. Sato, J. of Materials Sci., Volume 28 (1993), 1509-1514. 